\title{
Revista de Enfermagem

\section{RISCO DE QUEDAS EM IDOSOS: ESTRATÉGIA CUIDATIVA-EDUCACIONAL PARA CUIDADORES PARA ADOÇÃO DE MEDIDAS PREVENTIVAS}

\section{RISK OF FALLS IN THE ELDERLY: CARE-EDUCATIONAL STRATEGY FOR CARE GIVERS FOR THE ADOPTION OF PREVENTIVE MEASURES \\ RIESGO DE CAÍDAS EN ANCIANOS: ESTRATEGIA ATENCIÓN-EDUCATIVA DE CUIDADORES PARA LA ADOPCIÓN DE MEDIDAS PREVENTIVAS}

Daisy Teresinha Reis Coutinho ${ }^{10}$, Francisca Tereza de Galiza ${ }^{2}$, Jéssica de Menezes Nogueira ${ }^{3}$, Maria Vilani Cavalcante Guedes ${ }^{4}\left(\mathbb{0}\right.$, Odezio Damasceno Brito $^{5}$, Maria Célia de Freitas ${ }^{6}$ i]

\section{RESUMO}

Objetivo: implementar atividade educativa junto a cuidadores de idosos para prevenir quedas. Método: trata-se de uma pesquisa-cuidado, realizada em uma Instituição de Longa Permanência para Idosos, de abril a julho de 2019. Informa-se que participaram 23 cuidadores. Coletaram-se os dados em cinco encontros com intervenções educativas com análise fundamentada em autores estudiosos do assunto. Resultados: evidenciaram-se, nos encontros, os temas: As quedas causam muitos prejuízos para os idosos; O ambiente pode ser causador de quedas em idosos. Conclusão: permitiu-se, pela pesquisa-cuidado, uma favorável interação entre pesquisador-cuidador e pesquisados-cuidados, sensibilizando-os quanto à prevenção de quedas nos idosos residentes. Descritores: Cuidados de Enfermagem; Educação em saúde; Saúde do idoso institucionalizado; Idoso; Instituição de Longa Permanência para Idosos; Cuidadores.

\section{ABSTRACT}

Objective: to implement educational activity with caregivers of the elderly to prevent falls. Method: this is a care-research study, carried out in a Long-Term Care Institution for the Elderly, from April to July 2019. It is informed that 23 caregivers participated. Data was collected in five meetings with educational interventions with analysis based on authors who study the subject. Results: the following themes became evident during the meetings: Falls cause a lot of damage to the elderly; The environment can be a cause of falls in the elderly. Conclusion: the care-research allowed a favorable interaction between researcher-caregiver and researched-caregivers, making them aware of the prevention of falls in elderly residents.

Descriptors: Nursing Care; Health Education; Health of Institutionalized Elderly; Elderly; Long-Stay Institution for the Elderly; Caregivers. 


\section{RESUMEN}

Objetivo: implementar una actividad educativa con cuidadores de personas mayores para prevenir caídas. Método: se trata de una investigación asistencial, realizada en una Institución de Larga Estancia para Ancianos, de abril a julio de 2019. Se informa que participaron 23 cuidadores. Los datos se recolectaron en cinco reuniones con intervenciones educativas con análisis basados en autores académicos. Resultados: en las reuniones se destacaron los siguientes temas: las caídas causan mucho daño a los ancianos; El medio ambiente puede provocar caídas en los ancianos. Conclusión: la investigación asistencial permitió una interacción favorable entre investigadorcuidador y sujetos investigados-cuidados, sensibilizándolos sobre la prevención de caídas en ancianos residentes.

Descriptores: Atención de Enfermería; Educación en Salud; Salud del Anciano Institucionalizado; Anciano; Hogares para Ancianos; Cuidadores.

1,4,5,6 State University of Ceará/UECE. Fortaleza (CE), Brazil.

2,3 Federal University of Piauí/UFPI. Teresina (PI), Brazil.

${ }^{1}$ https: / / orcid.org/0000-0002-4989-9235

2 https: / / orcid.org/0000-0001-5217-7180

3 https: / / orcid.org/0000-0002-8201-7169

4 https: / / orcid.org/0000-0002-6766-4376

5 https: / / orcid.org/0000-0003-4008-3931

6 https://orcid.org/0000-0003-4487-1193

\section{Como citar este artigo}

Coutinho DTR, Galiza FT, Nogueira JM, Guedes MVC, Brito OD, Freitas MC. Risco de quedas em idosos: estratégia cuidativa-educacional para cuidadores para adoção de medidas preventivas. Rev enferm UFPE on line. 2021;15(2):e247773 DOI: https://doi.org/10.5205/1981-8963.2021.247773 
Sabe-se que o envelhecimento humano é um fenômeno e uma conquista mundial. Revelam-se, por dados das Nações Unidas, projeções de caráter global nas quais, entre 2019 e 2050, o número de pessoas com 65 anos ou mais, em todo o mundo, alcançará mais que o dobro; em contrapartida, o número de crianças menores de cinco anos permanecerá relativamente inalterado. ${ }^{1}$

Tem-se essa transição do perfil demográfico populacional caracterizado alterações epidemiológicas, com alta prevalência de doenças crônicas não transmissíveis, que podem levar a uma condição de incapacidade e evidenciar quadros de fragilidade.

Resulta-se a fragilidade, caracterizada como um evento multidimensional, da influência de condições biopsicossociais, ambientais, de alterações musculoesqueléticas e na função motora, as quais podem contribuir para um maior declínio funcional, a ocorrência de quedas, hospitalizações e institucionalização em Instituição de Longa Permanência para Idosos (ILPI). ${ }^{2}$

Tornam-se imprescindíveis, para o cuidado qualificado ao idoso, em especial, na ILPI, profissionais capacitados para avaliá-lo em todas as suas dimensões, desenvolvendo intervenções adequadas, fundamentadas em atividades de prevenção e promoção da saúde, com vistas a prevenir uma série de agravos e incapacidades relacionados ao desempenho das atividades de vida diária, a exemplo da prevenção de quedas.

Destaca-se, no contexto da institucionalização, a figura do cuidador de idosos, cujo compromisso é de auxiliar o idoso nas dificuldades diárias decorrentes do processo de envelhecimento, seja no domínio físico, psicológico, cognitivo ou social. Prevê-se, assim, que o cuidador é imprescindível para contribuir na qualidade de vida do idoso, desempenhando atividades de supervisão e auxílio na alimentação, higienização, medicação, mobilidade da pessoa idosa em atividades de recreação e lazer, entre outras. ${ }^{3}$

Desempenha-se papel fundamental, assim, pelos enfermeiros, ao desenvolverem práticas de educação em saúde junto aos cuidadores de idosos com vistas a suprir lacunas de conhecimento, suas expectativas e dúvidas. Realizam-se práticas educativas de Enfermagem, desde muito tempo, em todos os contextos de cuidado, especialmente, quando se tem, como foco, o ser humano, em particular, a pessoa idosa, representando um dos sustentáculos norteadores da prática do enfermeiro, devendo, como competência profissional, ser desenvolvidas nos diferentes cenários do cuidado, quer seja em atividades grupais ou individualizadas. ${ }^{4}$

Entende-se, portanto, que o processo educativo, por meio da pesquisa cuidado, permite o ensino-aprendizagem dos cuidadores para o cuidado e sensibiliza em relação à manutenção da capacidade funcional da pessoa idosa, habilitando-os sobre os riscos das condições de saúde do próprio idoso, bem como do ambiente. 


\section{OBJETIVO}

Implementar atividade educativa junto a cuidadores de idosos para prevenir quedas.

\section{MÉTODO}

Empregou-se, como metodologia, a pesquisa-cuidado. Transita-se este método nas relações interpessoais e intrapessoais, concretizando-se por meio das ações de cuidado. Deve-se, assim, a partir do momento que se identifica a situação problema vivenciada no grupo, algo ser planejado e realizado para transformar ou mudar a realidade. ${ }^{5}$

Desenvolveu-se a pesquisa em uma ILPI da cidade de Fortaleza (CE), no período de abril a julho de 2019. Identificaram-se idosos com o diagnóstico de Enfermagem risco de quedas após avaliação dos registros dos profissionais nos prontuários, bem como da anamnese de exame físico nos momentos de avaliação.

Detalha-se que participaram do estudo 23 cuidadores, trabalhadores da instituição, nos turnos manhã, tarde e noite.

Adotou-se como critério de inclusão: ser cuidador de idosos em risco de fragilização, definido por idoso capaz de gerenciar sua vida de forma independente e autônoma, todavia, encontrando-se em um estado dinâmico entre senescência e senilidade, resultando na presença de limitações funcionais (declínio funcional iminente), mas sem dependência funcional. Excluíram-se os cuidadores de férias e/ou de atestado.

Propõem-se, para viabilizar uma pesquisa-cuidado, cinco etapas: (1) Aproximação com o objeto de estudo; (2) Encontro com o ser pesquisado-cuidado; (3) Estabelecimento das conexões da pesquisa, teoria e prática do cuidado; (4) Afastamento do ser pesquisador-cuidador e ser pesquisado-cuidado e (5) Análise do apreendido. ${ }^{6}$

Realizaram-se, para a coleta de dados, cinco encontros educativos cuidativos-educacionais. Observa-se que estes ocorriam no período da tarde, às 17 horas, horário que não comprometia o atendimento dos idosos. Configuraram-se os encontros como espaços de troca e construção coletiva de saberes, de análise da realidade, de confrontação de experiências, de criação de vínculos socioafetivos e de exercício concreto dos direitos humanos. ${ }^{6}$

Optou-se pela roda de conversa pelo seu caráter educativo e reflexivo sobre as práticas cotidianas dos cuidadores dos idosos residentes e, ainda, pela possibilidade de maior aproximação dos cuidadores e informalidade. Propunham-se as rodas a habilitá-los na execução das atividades, observação atentiva dos idosos e do ambiente, sensibilizando-os quanto aos cuidados preventivos de quedas.

Organizaram-se cinco rodas de conversas, sendo quatro para a discussão das informações e a última para a avaliação do aprendizado. Acordaram-se todas as rodas com a gerente do serviço, 
que participou integralmente das atividades. Planejaram-se estas para atender aos horários dos cuidadores e não comprometer a rotina do serviço e, principalmente, o cuidado aos idosos. Acrescenta-se que cada roda de conversa durou, em média, 45 minutos.

Informa-se que todos assinaram o Termo de Consentimento Livre e Esclarecido (TCLE). Aprovouse o projeto pelo Comitê de Ética em Pesquisa da Universidade Estadual do Ceará sob o número de Parecer 3.153.552, de fevereiro de 2019.

Nominou-se a primeira roda de conversa como exercício de sensibilização para descontrair e envolver os cuidadores nas atividades propostas. Discutiram-se as formas como seriam desenvolvidas as atividades. Realizou-se um pré-teste com vistas a conhecer o que eles sabiam sobre os cuidados necessários à pessoa idosa, as alterações do processo de envelhecer, como prevenir as quedas dos residentes e a relação entre o grau de dependência e a sobrecarga de trabalho. Utilizou-se, para esse momento, recorte de figuras para conhecer sobre os temas, seguido de exposição e discussão. Explicitou-se, pelos participantes, após os recortes e as colagens, a tradução de suas figuras para todo o grupo.

Iniciou-se, no segundo momento, a roda de conversa com a exposição explicativa de dúvidas sobre as ideias e os conhecimentos revelados na oficina anterior. Elaborou-se um mapa de ideias com os termos mais frequentemente utilizados. Retomou-se o tema envelhecimento, suas alterações, especialmente, no caminhar. Pontua-se que a finalidade da discussão era refletir e reelaborar o conhecimento do grupo, socializando ideias, ações e situações vivenciadas pelos cuidadores em um cuidado comum. Representavam-se, pelos temas idosos, quedas, prevenção de quedas e cuidado com ambiente, abordados a partir de atividades em grupo, as experiências e observações cotidianas dos cuidadores.

Perguntou-se, no terceiro momento, sobre a prática diária na prevenção de quedas. Expuseramse, pelos participantes, suas experiências de cuidado e os modos possíveis das quedas acontecerem. Favoreceu-se, com isso, o preenchimento de lacunas identificadas na roda de conversa anterior, bem como o aprofundamento dos conhecimentos.

Confia-se que, nesse momento, os cuidadores, além de expressarem suas ideias, fortaleciam e corrigiam estratégias de cuidado aos idosos, em especial, para aqueles idosos em uso de bengalas, cadeira de rodas e/ou com dificuldades de deambular.

Realizou-se, no quarto encontro, uma vivência sobre "ser idoso" utilizando o jogo do envelhecimento no qual os cuidadores foram organizados em grupos para facilitar a atividade. Focava-se em fazê-los sentir as dificuldades vivenciadas pelo indivíduo no processo de envelhecimento. Utilizaram-se luvas para minimizar a destreza manual; óculos com filme sobre as lentes para simular dificuldade visual e pesos nas pernas como obstáculos para a deambulação. 
Distribuíram-se recipientes e receitas para o grupo com o objetivo de retirar os comprimidos dos frascos e sentir a dificuldade tanto do manuseio quanto da leitura. Destacava-se, pelos pesos, o comprometimento da mobilidade.

Solicitou-se que os mesmos caminhassem nos corredores da instituição, usando, ainda, tampões nos ouvidos, simulando dificuldade auditiva, e grãos de milho em um dos sapatos para simular problemas como dores na deambulação.

Questionaram-se os cuidadores, para analisar a efetividade do jogo, sobre a vivência ser idoso, as dificuldades e limitações identificadas. Expuseram-se, por cada grupo, suas experiências com comentários positivos e negativos sobre a velhice, tais como: dificuldades no tato, no caminhar, na leitura da receita, além das dores na mobilização ocasionada pelos pesos e grãos de milhos. Organizaram-se os referidos dados para discussão e análise na quinta roda de conversa.

Efetuou-se, na roda de conversa, quinta e última, a avaliação final que se destinou à exposição, pelos cuidadores, sobre as discussões e as vivências. Realizou-se uma exposição com diálogo e reflexão, sempre articulando o cuidado cotidiano e a realidade vivenciada pelos cuidadores no local. Organizaram-se os dados, as falas gravadas e transcritas, além dos registros escritos para análise em dois temas que foram validados pelos cuidadores no momento final: "As quedas causam prejuízos para os idosos" e "O ambiente pode ser causador de quedas em idosos".

\section{RESULTADOS}

Descreve-se que, dos 23 sujeitos do estudo, predominou o sexo feminino; a faixa etária entre 32 e 53 anos, com média 35 anos; o tempo de serviço mínimo de um ano. Identificaram-se, pelo pesquisador-cuidador, entre os pesquisados-cuidados, durante os momentos de conversas na roda, oito sem experiência no cuidado ao idoso, pois foram transferidos de outra instituição pública.

Caracteriza-se o cuidado a idosos com múltiplas comorbidades, para os pesquisados-cuidados, cuidadores, com apenas um ano de trabalho na instituição, como atividade difícil, delicada e estressante e, em algumas situações, esse cuidado os deixava preocupados e questionando as mudanças decorrentes do envelhecimento; já para os pesquisadores-cuidadores, com tempo de serviço maior que um ano, os idosos são pessoas delicadas e requerem muita atenção para a sua situação não complicar e deixá-los acamados.

\section{DISCUSSÃO}

\section{As quedas causam muitos prejuízos para os idosos}

Assinala-se que as rodas de conversa para a sensibilização dos cuidadores tiveram, como foco, a atenção aos idosos e a adoção de medidas preventivas de quedas. Discutiram-se tais medidas nos encontros e, especialmente, na prática de cuidador. Percebe-se que uma das vantagens no uso do 
método de pesquisa-cuidado é a possibilidade de implementar cuidados imediatamente após a identificação da necessidade de cuidar. ${ }^{7}$

Caracterizam-se, nas falas dos pesquisados-cuidados, a preocupação com a prevenção de quedas e o ensino dos efeitos da queda sobre o idoso, ampliando a responsabilidade, pois desejam e se preocupam com a manutenção da mobilidade do idoso. Coincide-se tal atitude com a afirmação: “Cuidar de um idoso vai muito além da conduta terapêutica. É estar atento à qualidade de vida, aos níveis de autonomia e independência, ao meio ambiente social e físico. É estar comprometido com a promoção da saúde, com a prevenção de enfermidades, assim como com o tratamento adequado de quaisquer condições vigentes, nesse caso, inclui-se a prevenção de quedas, que geram inúmeros adoecimentos aos idosos". ${ }^{8-9}$

Associava-se, pelos cuidadores, com sua maneira própria de captar e de compreender a realidade, a necessidade do cuidado preventivo com o autobenefício de não aumentar as demandas de cuidado para eles, do mesmo modo que a compreensão de algo corresponde, cedo ou tarde, a uma ação, a uma compreensão, primordialmente, a atitudes que podem modificar a prática cotidiana do cuidado. ${ }^{10}$

Relatou-se, pelos cuidadores, que alguns idosos apresentam dificuldade quanto à memória, atenção e linguagem, dificuldade para caminhar nos corredores, bem como firmar-se para fazer as atividades pessoais. Nota-se, progressivamente, à medida que aumentam a debilidade e a dependência do idoso, que os encargos do cuidar poderão sofrer novas mudanças, exigindo maiores esforços para suprir as necessidades que acompanham a diminuição da capacidade funcional do idoso. Alerta-se, como resultado das repercussões do cuidar e do estado de vulnerabilidade ao qual - cuidador se expõe, que podem surgir desgastes físicos, psicológicos e/ou sociais e, consequentemente, gerar sobrecarga de trabalho, particularmente, quando o idoso cai e compromete a mobilidade, aumentando a demanda de cuidado. ${ }^{11}$

Expôs-se, nesse contexto, a necessidade de aprender estratégias preventivas de quedas para minimizar a sobrecarga de trabalho cotidiano da equipe. Referiu-se que os idosos mais dependentes requerem maior esforço físico e maior demanda mental pelo detalhamento do trabalho, diferentemente daqueles que se locomovem com independência, mesmo em cadeira de rodas.

Caracteriza-se a dependência física do idoso por dificuldade ou incapacidade de deambular, diminuição de mobilidade ou incontrolabilidade de movimentos, causando perda de autonomia e necessidade constante de auxílio de seus cuidadores. Acrescenta-se que esses, por sua vez, demandam de mais trabalho dos cuidadores para atender às suas necessidades, gerando, em muitos casos, um sentimento de tristeza e depressão. ${ }^{12}$ 
Pontua-se que, nos idosos, as quedas podem comprometer a capacidade funcional, resultando em perda de habilidades para realizar as atividades da vida diária. Sobrecarrega-se, pelo comprometimento na realização dessas atividades, consequentemente, a equipe de saúde, em especial, os cuidadores responsáveis pela higiene, alimentação e banho de sol. Dever-se-á o cuidador, no que se refere ao idoso frágil, aquele impossibilitado de colaborar com o cuidado, realizar atentivamente a higiene de modo seguro, prevenindo qualquer dano ao idoso.

Percebeu-se, sobre as atividades desenvolvidas, que, a cada encontro, os próprios cuidadores identificavam as fragilidades e lacunas no cuidado prestado, pois eles pensavam e apontavam novas estratégias para o grupo a partir das suas próprias descobertas e reflexões de suas experiências cotidianas.

Abordou-se, ainda, que, na instituição, a maioria dos idosos tem prescrições de vários medicamentos, seja por causa das múltiplas comorbidades ou devido às agitações e/ou inquietações apresentadas por eles, outro fator de preocupação com quedas. Favorecem-se, pelo uso continuado de medicamentos do tipo sedativo, a ocorrência e o risco de reações adversas, pondo em risco, ainda, os idosos em condições de adoecimento.

Infere-se que tais eventos podem aumentar de acordo com a dose utilizada, o tempo de uso, a associação com outros fármacos e por influência da farmacocinética e farmacodinâmica alterada pelo processo de envelhecimento e as prescrições de tais drogas para os idosos, quando necessárias, devem ser monitoradas criteriosamente. ${ }^{13}$

Despertou-se, neste caso, para os riscos, em particular, das quedas, devido aos efeitos dos medicamentos usados pelos idosos, os benzodiazepínicos, principalmente por aqueles idosos com quadros demenciais confirmados, agitação psicomotora, agressividade, inquietação, dentre outros.

Revelou-se, pela demanda de informação requisitada ao pesquisador-cuidador, no momento das atividades, a existência de inúmeras dúvidas dos sujeitos do estudo, pois foram identificadas lacunas no conhecimento dos mesmos, tanto em relação ao cuidado efetivo quanto no que se refere ao uso de medicamentos, seus efeitos e à necessidade do olhar atentivo aos idosos residentes.

Ilustra-se esse processo, nas oficinas, devido aos cuidadores acrescentarem, ainda, que a vigilância contínua aos idosos seja necessária, principalmente daqueles que usam a medicação de maneira continuada. Apontou-se, por outros, a extrema necessidade no uso do medicamento para que o idoso possa manter o repouso adequado e tornar possível a realização do cuidado. Citou-se, no entanto, que os cuidados serão enfatizados, mas que o ambiente, os calçados dos idosos e a própria velhice são fatores interessantes para a observação. Afirmou-se que a atividade de cuidado realizada por eles é delicada e extremamente responsável, uma vez que são pessoas que, na ausência de atenção, estão expostas a riscos. 
Averiguou-se, pelos resultados das atividades com os cuidadores, a necessidade de educação permanente para refletirem sobre as atividades cotidianas, oriundas da prática, com a finalidade de melhorar a qualidade do cuidado, bem como a qualidade de vida dos idosos residentes, pelo comprometimento, conscientização e responsabilidades dos cuidados oferecidos. Percebeu-se que, a cada encontro, outras reflexões aconteciam e construíam-se novas estratégias para o grupo.

Verifica-se que um dos resultados mais atenciosos das pessoas comprometidas com o processo de ensino-aprendizagem é que, junto com novos conhecimentos, provoca-se, nos envolvidos, a aparição de novas formas de pensar e de atuar, que geram novos recursos e buscam solucionar novos questionamentos e problemáticas na sociedade. ${ }^{14}$

Sabe-se que o processo cuidativo-educativo é um curso de ação sistemática, sequencial, lógica, planejada e com base científica, que consiste em duas operações interdependentes principais; o ensino e a aprendizagem. Forma-se, nesse processo, um ciclo contínuo que também envolve dois agentes interdependentes, o educador e o aprendiz, que, juntos, desempenham seus papéis, cujo resultado leva a mudanças de comportamento mutuamente desejado. ${ }^{15}$

\section{O ambiente pode ser causador de quedas em idosos}

Constata-se que os cuidadores também pensaram no ambiente, especialmente, quando não é bem cuidado, esclarecendo dúvidas sobre como fazer para oferecer o ambiente que propicie, ao idoso, segurança porque, às vezes, o cuidado focaliza os idosos mais frágeis e aqueles que deambulam podem cair. Acredita-se que as respostas em relação às dúvidas existentes foram ricas e esclarecedoras. Afirmou-se que desconheciam os riscos de quedas, principalmente, porque não conheciam as alterações orgânicas decorrentes do processo de envelhecimento, que, associadas a questões ambientais, favorecem a ocorrência de quedas. Serviu-se tal atividade para partilhar conhecimentos conforme a realidade, favorecendo o reconhecimento de práticas incorretas e a reflexão de novas atitudes necessárias aos cuidados dos idosos.

Pode-se o ambiente da ILPI onde o idoso se encontra inserido apresentar-se como importante fator de quedas quando se encontra precário. Torna-se fundamental, assim, a promoção de ambiente seguro, especialmente, nos casos em que há instabilidade postural do idoso, destacandose que os fatores ambientais, aliados às condições funcionais e de mobilidade dos idosos, influenciam a ocorrência de quedas. ${ }^{17}$

Torna-se indispensável atentar para algumas particularidades que favorecem um ambiente de ILPI mais seguro e acolhedor com vistas a promover conforto, autonomia e prevenir quedas como: pisos antiderrapantes; camas com grades laterais; presença de barras de apoio em corredores e banheiros; iluminação adequada, especialmente, à noite; mobílias colocadas em locais que não dificultem o caminho do idoso; não usar tapetes; banheiros com portas amplas e sanitários com 
altura adequada e barras laterais; cadeiras com apoio para braços e altura apropriada; corredores livres de obstáculos.

Enfatizou-se, pelos cuidadores, a deficiente infraestrutura dos ambientes internos e externos da instituição em relação ao piso escorregadio, desnível de piso, cadeiras sem pés antiderrapantes, ausência de barras de apoio em corredores e quartos.

Registra-se, portanto, que os locais com maior índice de quedas são o quarto, seguido de banheiros. Salienta-se que tal fato possa decorrer da ausência de estrutura física de muitos destes ambientes, os quais não possuem piso antiderrapante, barras de apoio e luz noturna adequada, por exemplo. Revela-se, por evidências cientificas, que, entre idosos residentes em ILPIs, o quarto e o banheiro correspondem, juntos, a 75\% dos locais de ocorrência de quedas, sendo o quarto o local predominante..$^{18-19}$

Acredita-se que assegurar espaço seguro perpassa aspectos que envolvem a delegação de tarefas a outros profissionais que possibilitem identificar práticas errôneas e lidar com eventos nos quais a segurança possa estar prejudicada. Constata-se que o cerne do cuidado com o ambiente para a prevenção das quedas é a meta primordial na promoção da saúde do idoso e, nesse contexto, é imprescindível agregar o conceito de acessibilidade na prática de cuidado do enfermeiro e demais profissionais, com vistas a um trabalho multiprofissional. ${ }^{20}$

Envolvem-se, pela importância de monitorar os fatores extrínsecos ao risco de quedas a partir do ambiente físico até materiais e inúmeros dispositivos de auxílio, bem como a conservação destes em adequado estado de uso, procedimentos desde a aquisição, utilização e manutenção periódica dos mesmos. Destaca-se, exemplificando o exposto e a relação com as quedas em idosos residentes de ILPIs, o uso equivocado de auxiliares de deambulação, por vezes, inapropriados ao modo de deambular do idoso ou até mesmo por apresentarem-se danificados. ${ }^{20}$

Infere-se, igualmente, que o uso de calçados impróprios, como chinelos e sapatos com solado de borracha ou saltos, mal ajustados aos pés dos idosos, dificultando a deambulação e, em alguns casos, em associação com pisos escorregadios e desnivelados, pode predispor a tropeços e, consequentemente, a quedas. Considera-se que as ILPIs aceitam doações de vestuário e calçados e que, muitas vezes, não são adequados ou na medida dos idosos, o que salienta a relevância de uma seleção destes itens como forma de prevenção, visto que os mesmos devem contribuir para a segurança. ${ }^{20-21}$

Informa-se, neste sentido, que os cuidadores refletiram e expuseram o planejamento de estratégias que poderiam alterar os fatores de risco modificáveis, estabelecendo ações de cuidado de vigilância continuada e cuidados específicos relacionados ao ambiente. 
Podem-se perceber, embora esta intervenção educativa tenha sido desenvolvida em cinco oficinas, configurando-se uma possível limitação do estudo, mudanças entre os cuidadores de idosos no tocante ao posicionamento de antes e o atual, ao reelaborarem seus conceitos e conhecimentos acerca do cuidado implementado ao idoso residente em relação à prevenção de quedas.

\section{CONCLUSÃO}

Permitiu-se, pela experiência com a roda de conversa, partilhar um contínuo de troca, integração e aproximação entre o pesquisador-cuidador e os pesquisados-cuidados. Entende-se que o conhecimento da realidade de trabalho com os idosos e dos modos das possíveis quedas permitiu um movimento de mudanças e a análise sobre as atividades de cada um em ralação aos idosos.

Pode-se considerar, como limitações, a não permanência no dia a dia dos cuidadores, visto que o cuidado precisa de constante atenção para não incorrer em novos problemas.

Favoreceu-se, também, pela pesquisa-cuidado, a abertura para discussões sobre os problemas existentes na instituição, em relação a outros fatores relacionados ao cuidado aos idosos, apontando para novos momentos para o processo educativo com os cuidadores para aprimorar os cuidados não apenas na prevenção de quedas, mas identificando outros problemas correlatos.

\section{CONTRIBUIÇÕES}

Acredita-se que esta pesquisa fornece resultados importantes para o cuidado de saúde do idoso e a aproximação do cuidador na prevenção de quedas em Instituições de Longa Permanência para Idosos.

\section{CONFLITO DE INTERESSES}

Nada a declarar.

\section{REFERÊNCIAS}

1. United Nations, Department of Economic and Social Affairs, Population Division (2019). World Population Prospects 2019: Highlights. ST/ESA/SER.A/423. Available from:

\section{https://population.un.org/Files/WPP2019/Highlights}

2. Carneiro JA, Lima CA, Costa FM, Caldeira AP. Cuidados em saúde estão associados à piora da fragilidade em idosos comunitários. Revista de Saúde Pública [Internet]. 2019 [acesso em 15 mai. 2020]; 53:32. DOI: https://doi.org/10.11606/s1518-8787.2019053000829.

3. Souza G, Duarte M, Vieira P, Albuquerque K, Evangelista C, Silva M, Figueiredo M. Family assessment of elderly patients with liver disease. Journal of Nursing UFPE on line [internet]. 2018 [acesso em 27 mar. 2020]; 12(10):2690-2702. DOI: https://doi.org/10.5205/1981-8963- 
4. Santos JMS, Ribeiro LCRB, Lopes RF. Ações educativas em instituições de longa permanência para idosos: uma revisão narrativa da literatura. Brazilian Journal of Development. [Internet]. 2020

[acesso em 12 mai. 2020]; 6(3):12140-12152. DOI: https://doi.org/10.34117/bjdv6n3-177

5. Lacerda MR, Costenaro RGS (Org). Metodologias da Pesquisa para Enfermagem e Saúde: da teoria à prática. 1ed. Porto Alegre: Moriá; 2016.

6. Candau, V. M. F., \& Sacavino, S. B. Educação em direitos humanos e formação de educadores.

Educação (Porto Alegre, impresso). [Internet]. 2013 [acesso em 27 fev. 2020]; 36(1), 59-66.

Available from: https: //www.redalyc.org/pdf/848/84825694009.pdf.

7. Pontes KMA, Silva LF. Implementação do método pesquisa-cuidado com base na teoria do conforto: relato de experiência. Ciência, Cuidado e Saúde. [Internet]. 2014 [acesso em 19 abr. 2020]; 13(2):388-393. DOI: https://doi.org/10.4025/cienccuidsaude.v13i2.16439

8. Aires M, Fuhrmann AC, Mocellin D, Dal Pizzol FLF, Sponchiado LF, Marchezan CR, Bierhals CCBK, Day CB, Santos NO, Paskulin LMG. Sobrecarga de cuidadores informais de idosos dependentes na comunidade em municípios de pequeno porte. Rev Gaúcha Enferm. [Internet]. 2020 [acesso em 01 jun. 2020]; 41 (esp):e20190156. DOI: https://doi.org/10.1590/1983-1447.2020.20190156.

9. Ximenes MAM, Fontenele NAO, Bastos IB, Macêdo TS, Galindo Neto NM, Caetano JA, Barros LM. Construção e validação de conteúdo de cartilha educativa para prevenção de quedas no hospital. Acta paul. enferm. [Internet].2019 [acesso em 21 abr. 2020]; 32(4):433-441. DOI:

https://doi.org/10.1590/1982-0194201900059.

10. Nogueira IS, Acioli S, Carreira L, Baldissera VDA. Atenção ao idoso: práticas de educação permanente do Núcleo de Apoio à Saúde da Família. Rev. esc. enferm. USP [Internet]. 2019 [acesso em 20 abr. 2020]; 53:e03512. DOI: https://doi.org/10.1590/s1980-

\section{$\underline{220 \times 2018022103512 .}$.}

11. Silva JCS, Souza FDCR, Aoyama EA. A Incidência do uso indiscriminado de medicamentos. ReBIS [Internet]. 2020 [acesso em 28 abr. 2020]; 2(1):95-9. Available from: http://revista.rebis.com.br/index.php/rebis/article/view/360/127.

12. Bispo NNC, Gonçalves FF, Costa VSP, Lodovici FMM, Lopes RGC, Concone MHVB. O impacto da doença na vida cotidiana em pessoas idosas institucionalizadas. Investigação Qualitativa em Saúde. [Internet]. 2019 [acesso em 30 mai. 2020]; CIAIQ2019, 2, 1292-1302. Available from: https://proceedings.ciaiq.org/index.php/CIAIQ2019/article/view/2276/2197.

13. Costa JB, Furtado LGS, Girard CCP. Saberes e Práticas do Enfermeiro na Consulta com o idoso na Estratégia Saúde da Família. Rev. Aten. Saúde. [Internet]. 2019 [acesso em 12 fev. 
14. Dantas DG, Port KDSCM, Batista JPS, Mendes CMM, Ribeiro IAP, Marques LL. Uso de Psicofármacos por idosos institucionalizados: Aspectos epidemiológicos e frequência de queda. Revista Enfermagem Atual [Internet]. 2019 [acesso em 21 abr. 2020]; 89(27). Available from: https://revistaenfermagematual.com.br/index.php/revista/article/view/463.

15. Mendes TMC, Ferreira TLS, Carvalho YM, Silva LG, Souza CMCL, Andrade FB (2020). Contribuições E Desafios Da Integração Ensino-Serviço-Comunidade. Texto contexto-enferm. [Internet]. 2020 [acesso em 21 abr. 2020]; 29: e20180333. DOI: https://doi.org/10.1590/1980-265xtce-2018-0333.

16. Gautério DP, Zortea B, Santos SSC, TaroucoBS, Lopes MJ, Fonseca CJ. Risk Factors for new accidental falls in elderly patients at Traumatology Ambulatory Center. Invest Educ Enferm. [Internet]. 2015 [acesso em 23 abr. 2020]; 33(1):35-43. Available from:

http: / / www.scielo.org.co/scielo.php?script=sci_arttext\&pid=S012053072015000100005\&lng=en\&tln $\mathrm{g}=\mathrm{en}$.

17. Ferreira LMBM, Ribeiro KMOBF, Jerez-Roig J, Araújo JRT, Lima KC. Quedas recorrentes e fatores de risco em idosos institucionalizados. Ciênc. saúde coletiva [Internet]. 2019 [acesso em 24 abr. 2020]; 24(1):67-75. DOI: https://doi.org/10.1590/1413-81232018241.35472016.

18. Büchele G, Becker C, Cameron ID, König HH, Robinovitch S, Rapp K. Predictors of serious consequences of falls in residential aged care: analysis of more than 70,000 falls from residents of Bavarian nursing homes. J Am Med Dir Assoc [Internet]. 2014 [acesso em 28 abr. 2020]; 15(8):559563. DOI: https://doi.org/10.1016/j.jamda.2014.03.015.

19. Baixinho CRSL, Dixe MACR, Henriques MAP. Queda nas Instituições de Longa Permanência para Idosos: validação de protocolo. Rev. Bras. Enferm. [Internet]. 2017 [acesso em 26 abr. 2020]; 70(4):740-746. DOI: https://doi.org/10.1590/0034-7167-2017-0109.

20. Rosa VPP, Cappellari FCBD, Urbanetto JS. Analysis of risk factors for falls among institutionalized elderly persons. Rev. bras. geriatra. gerontol. (Online) [Internet]. 2019 [acesso em 09 dez. 2019]; 22(1):e180138. DOI: http://dx.doi.org/10.1590/1981-22562019022.180138.

21. Alves MB, Menezes MDRD, Felzemburg RDM., Silva VAD, Amaral JBD. Long-stay institutions for the elderly: physical-structural and organizational aspects. Escola Anna Nery. [internet]. 2017 [acesso em 21 out. 2020]; 21(4):e20160337. DOI: https://doi.org/10.1590/2177-9465-EAN-20160337.

\section{Correspondência}

Odézio Damasceno Brito

E-mail: odeziod@gmail.com 
Copyright $\odot 2021$ Revista de Enfermagem UFPE on line/REUOL.

Este é um artigo de acesso aberto distribuído sob a Atribuição CC BY 4.0 Creative Commons AttributionShareAlike 4.0 International License, a qual permite que outros distribuam, remixem, adaptem e criem a partir do seu trabalho, mesmo para fins comerciais, desde que lhe atribuam o devido crédito pela criação original. É recomendada para maximizar a disseminação e uso dos materiais licenciados. 